\title{
Electrical stunning of fish: the relationship between the electric field strength and water conductivity
}

\author{
Jeff Lines $^{\mathrm{a}, *}$, Steve Kestin ${ }^{\mathrm{b}, 1}$ \\ ${ }^{a}$ Silsoe Research Institute, Wrest Park, Bedford, MK45 4HS, United Kingdom \\ ${ }^{\mathrm{b}}$ Department of Clinical Veterinary Science, University of Bristol, Langford Bristol, BS40 7EU, United Kingdom
}

Received 5 April 2004; received in revised form 16 July 2004; accepted 21 July 2004

\begin{abstract}
The electric field in water required to stun trout is investigated experimentally. The results show how the electric field depends on water conductivity. The electric fields in water required to generate permanent insensibility in trout following exposure durations of 15,30 and $60 \mathrm{~s}$ are identified. A mathematical model of the electric field in the stunning tank is constructed and shown to produce results consistent with the experimental results. This simulation indicates that the electric field that occurs in the fish will vary little with variation of the fish position in the tank and fish size but that there many be a significant variation in the electric field experienced by individual fish in a high density cluster of fish if water conductivity is below $50 \mu \mathrm{S} / \mathrm{cm}$ or greater than $500 \mu \mathrm{S} / \mathrm{cm}$.
\end{abstract}

(C) 2004 Elsevier B.V. All rights reserved.

Keywords: Welfare; Trout; Slaughter; Electric stunning; Water conductivity

\section{Introduction}

Electric stunning in water can be an efficient and humane slaughter method for fish. Its welfare benefits include the very rapid transition to insensibility, which is generally associated with electric stunning and that the fish need not be singulated, restrained,

* Corresponding author. Tel.: +44 1525 860000; fax: +44 1525861735.

E-mail addresses: jeff.lines@bbsrc.ac.uk (J. Lines), steve.kestin@bristol.ac.uk (S. Kestin).

1 Tel.: +44 177 9289313; fax: +44 1179289324 . 
touched or removed from water before they are insensible (Robb and Kestin, 2002). Application of the stun is also reliable and can be achieved at high speed since it requires no moving parts and no targeting of the fish.

This paper focuses on the electric field (or voltage gradient) in water required to stun portion sized rainbow trout (Oncorhynchus mykiss). Regulations and guidelines governing electrical stunning of most animals define the electrical requirements in terms of the current required rather than the voltage. However, specification of current is unsuitable for fish because, being surrounded by a conducting medium, the electrical current can pass through any part of the fish body or around it.

Humane slaughter is achieved if the fish becomes insensible very soon after exposure to an electric field and remains insensible until death occurs. Death may occur as a result of deliberately bleeding the fish, due to asphyxiation or as a direct result of the electric stun. The lower limit of the electric field strength, which may be used is determined by the requirement to create rapid insensibility. Exposure to an electric field that is too weak may be expected to result in paralysis rather than insensibility. The upper limit on the electric field is determined by the need to avoid carcass damage such as haemorrhages and broken bones, and also by the rapidly increasing electrical power requirement. Between these two bounds there can exist a range of acceptable electric fields which, with a suitable exposure duration, can result in permanent insensibility.

Behavioural indicators of insensibility have been investigated by Kestin et al. (2002) and by Robb and Roth (2003). Observations of brain function using visually evoked responses (VER) have shown that the return of normal brain function following an electric stun is well correlated with the return of normal periodic motion of the gill covers and the eye roll reflex. These simple behavioural tests have been used to determine the state of sensibility of the experimental fish in this work. This approach has been used previously by Roth et al. (2003) and Robb et al. (2002). Unfortunately, the onset of insensibility at the beginning of exposure to an electric field is not observable by this method since the muscle tension caused by the electric field stiffens the body and flares the gill covers. Experiments have however shown that after a 1-s exposure to an electric field of $2.3 \mathrm{~V} / \mathrm{cm}$ almost all trout remained motionless and apparently insensible for several seconds. This suggests that the onset of insensibility is occurring within less than $1 \mathrm{~s}$ (Lines et al., 2003).

Carcass damage is frequently associated with electric stunning (Sharber and Carothers, 1990; Roth et al., 2003). Most authors agree that carcass damage is primarily related to electrical wave form and electric field strength (Lamarque, 1990; Spencer, 1967; McMichael, 1993). The incidence of haemorrhages and broken bones in electrically stunned trout was investigated by Robb et al. (2002). This showed carcass damage during electric stunning was influenced by the frequency of the alternating electrical current, and that damage free electric stunning could be achieved by the use of a $1000-\mathrm{Hz}$ electric field. Extended exposure to an electric field was shown to result in no increase in carcass damage.

The conductivity of river water varies widely. In the UK, trout are farmed in water ranging in conductivity from below 40 to over $700 \mu \mathrm{S} / \mathrm{cm}$. Differences in water conductivity lead to differences in the voltage and current which need to be applied to the water in order to stun the fish. An understanding of the effect of water conductivity is needed to enable electric stunning devices to be developed which are suitable for use in a 
wide range of waters. Recent work showed that a 60 -s exposure to a $1000-\mathrm{Hz}$ electric field of $2.5 \mathrm{~V} / \mathrm{cm}$ would create permanent insensibility in portion sized trout in water of conductivity 300-600 $\mu \mathrm{S} / \mathrm{cm}$ (Lines et al., 2003). It was not known how this work should be applied in trout farming areas of low or high conductivity water.

The work reported in this paper explores the effect of water conductivity on the electric field required to stun trout. It was motivated by the need to define electrical field requirements for waters in a wide conductivity range, and also by the wish to start to investigate the implications of this research for stunning fish in sea water (which has a conductivity of around $50,000 \mu \mathrm{S} / \mathrm{cm}$ ). Experimental results are reported together with analysis of the data. A predictive model of the electric field is constructed and used to interpret the experimental data and to extrapolate beyond the available data. It does not discuss how electric stunning can be applied in practice. This subject is covered by Lines et al. (2003) and by Lines and Kestin (2004a,b).

All the experiments and analysis are based on a system where the electric field in the water is uniform (i.e. the electrodes are parallel and extend the full width and height of the water), where the fish are orientated so their head-tail axis is perpendicular to the direction of the electric field, and where the electric field is generated using $1000-\mathrm{Hz}$ sinusoidal voltage. All quoted voltages and electric fields etc refer to rms values.

The experimental work reported in this paper was done in accordance with the UK laws relating to experimentation on animals and the experiments were designed to minimise suffering. No fish were used in experiments more than once and all fish that showed signs of recovery from the stun were quickly killed either by a blow to the head or by exposure to an electric field known to be lethal.

\section{Experimental technique}

The fish used in this experiment were commercially farmed rainbow trout, which had reached harvest size. They had a mean mass of $384 \mathrm{~g}$ and individual fish had a standard deviation around this mean of $60 \mathrm{~g}$. The trout were housed in a concrete raceway in farm water of conductivity $500 \mu \mathrm{S} / \mathrm{cm}$ prior to slaughter.

The stunning tank was cuboid, $40 \mathrm{~cm}$ long, $15 \mathrm{~cm}$ wide and with a water depth of approximately $15 \mathrm{~cm}$. Sheet electrodes covered the two large sides of the tank. These were powered by an amplifier drawing its signal from a sinusoidal signal generator. The tank was filled with oxygenated de-ionised water and small quantities of salt $(\mathrm{NaCl})$ were added to increase the conductivity to the required level. Water conductivity measurements were made by direct measurement of the water depth, tank dimensions and of the voltage and current passing through the tank in the absence of fish.

The trout were removed from the concrete raceway using a net, and placed in a small tank of oxygenated, de-ionised water for a few seconds to remove the farm water. They were then transferred in groups of five to the slaughter tank. The trout were orientated parallel to the electrodes by the restricted width of the tank. A few seconds after placing the fish in the slaughter tank, a $1000-\mathrm{Hz}$ sinusoidal voltage was applied across the electrodes for the required duration. The fish were then transferred to farm water in an observation tank where they were monitored for $5 \mathrm{~min}$ for the return of regular opercular 
movement. Fish that recovered this reflex were deemed to have recovered sensibility. Those that failed to recover regular opercular movement within 5 min were deemed to be permanently insensible. Where there was doubt about the regularity of the opercular movement, the fish were examined for the eye roll reflex. All fish that recovered sensibility were killed humanely as soon as was practical.

Trout were stunned using the following parameters:

Electric field in water

Exposure duration

Nominal water conductivity

Electric field frequency
$1.1-6.3 \mathrm{~V} / \mathrm{cm} \mathrm{rms}$

15,30 and $60 \mathrm{~s}$

$50,100,160,300,500$ and $1000 \mu \mathrm{S} / \mathrm{cm}$ $1000 \mathrm{~Hz}$ sinusoidal

Measurements were also made of the electrical conductivity of recently killed trout. The fish were placed in water of various conductivities with an electric field across it. The voltage across the flanks of the fish was compared with the voltage, which existed between the same points when the fish was removed. The fish conductivity was considered to be equal to the conductivity of the surrounding water when these voltages were equal.

\section{Experimental results}

Experimental results relating to a 30 -s exposure are presented graphically in Fig. 1. It shows the proportion of trout in each group, which did not recover sensibility within 5 min following removal of the electric field. This parameter is, for convenience, termed the mortality within the group. Each point in the figure represents a group of at least 20 fish, with the exception of the three identified points, which represent 9 or 12 fish. This figure illustrates the range of effect that a single value of electric field in the water can have within the range of conductivities associated with fresh river water in the UK. These

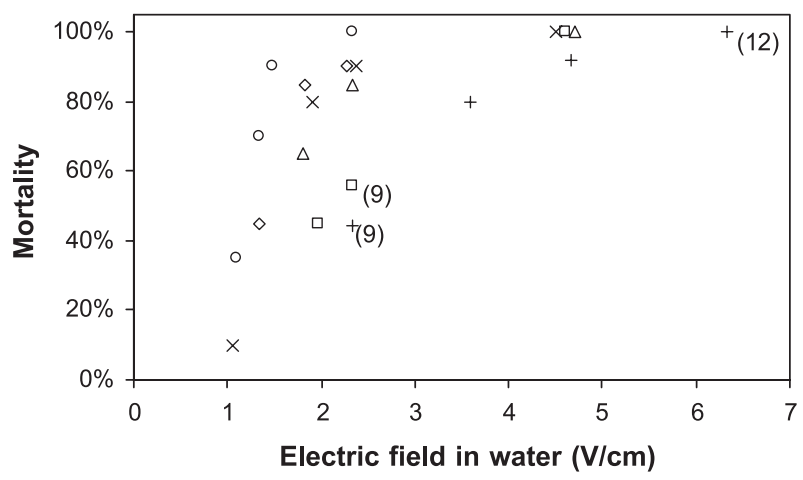

Fig. 1. Mortality in groups of fish after a 30-s exposure to an electric field in water of various conductivities. Points represent groups of at least 20 fish except those marked (9) or (12), which represent 9 or 12 fish, respectively. The symbols $+, \square, \triangle, \times, \diamond$ and $\bigcirc$ indicate water conductivities of 50,100,160,300, 500 and $1000 \mu \mathrm{S} / \mathrm{cm}$, respectively. 


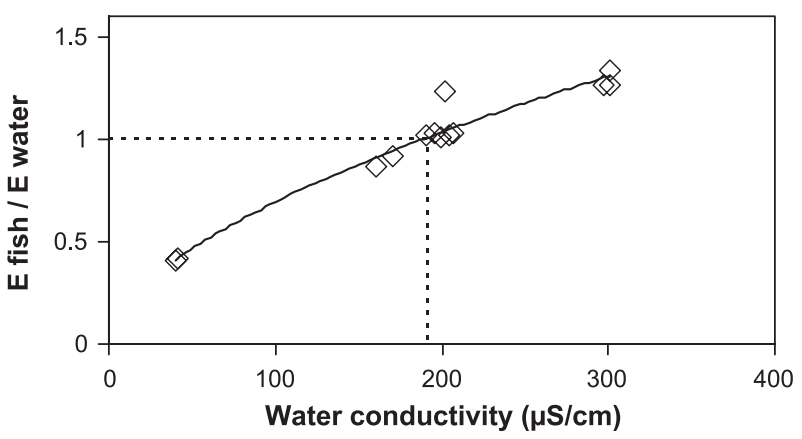

Fig. 2. Experimental measurements of the electric field across the flanks of a trout and the electric field in the water at the same location without the trout in water of different conductivities.

experimental results together with results of exposing the trout to an electric field for 15 and $60 \mathrm{~s}$ are tabulated in Appendix A.

Fig. 2 shows the ratio of the measured electric field across the flanks of a dead trout to that measured in the water for a range of water conductivities. It indicates that the electrical conductivity of the trout was $190 \mu \mathrm{S} / \mathrm{cm}$. Similar measurements with four other fish suggested a range of conductivities from 190 to $240 \mu \mathrm{S} / \mathrm{cm}$.

\section{Analysis of data}

To interpret this data the following assumptions are made:

- that the electric field in the water produces an electric field in the fish which is proportional to the electric field in the water and that the constant of this proportionality is a function of the water conductivity.

- that for a given period of exposure and within the population of fish used in the experiment, the electric field in the fish which results in permanent insensibility is a normally distributed value.

Based on these assumptions, values for the constants of proportionality and the parameters of the normal distribution have been estimated by transforming the data and fitting it to a normal distribution curve as described below.

Arbitrary values were first assigned to the ratios between the electric field in the water and the electric field in fish for water conductivities of 50, 100, 160, 300, 500 and $1000 \mu \mathrm{S} / \mathrm{cm}$. These values were constrained by the requirement that the ratio should be unity at a water conductivity of $190 \mu \mathrm{S} / \mathrm{cm}$. Linear interpolation was used to calculate the ratios at water conductivities between the values, which were defined. These ratios were used to calculate the electric field in the fish for each electric field and water conductivity. Following this data transformation and with the correct values of the ratios, we would expect the percentage mortality associated with each value of electric field in the fish to lie on a cumulative normal distribution curve. 


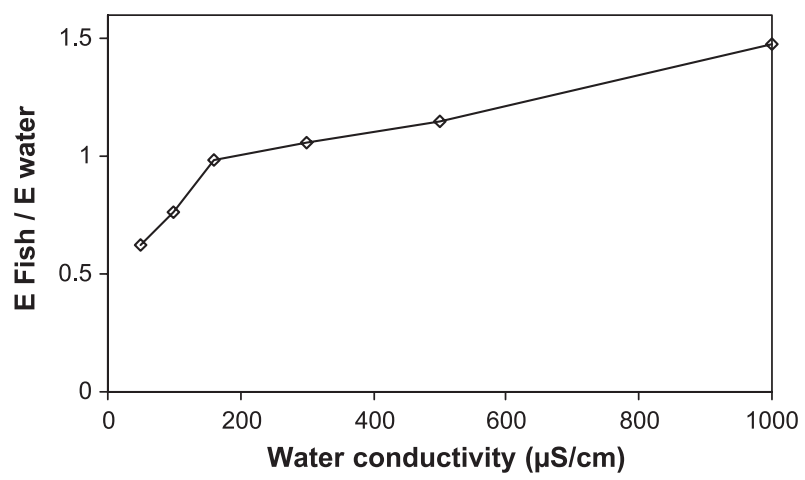

Fig. 3. Constant of proportionality between the electric field in the fish and the electric field in waters of various conductivities derived from experimental data.

An iterative minimisation routine was used to identify the electric field ratios at the six defined conductivities and the distribution mean and standard deviation which minimised the sum square difference between the experimentally observed mortality rates and the normal distribution curve.

This minimisation resulted in the relationship between the electric field in the water and the electric field in the fish given in Fig. 3. The transformed data values and the normal distribution curve to which they are fitted are shown in Fig. 4. This normal distribution has a mean of $1.63 \mathrm{~V} / \mathrm{cm}$ and a standard deviation of $0.575 \mathrm{~V} / \mathrm{cm}$. The degree with which this data fits to the normal distribution curve provides support for the assumptions made in this analysis.

In determining the parameters to be used for humane slaughter, we aim to generate permanent insensibility in the whole population. In order to do this, it is reasonable to select the electric field in the fish equivalent to the mean plus three standard deviations, since this should result in permanent insensibility for $99.7 \%$ of the fish. This experiment points to a target electric field of $3.36 \mathrm{~V} / \mathrm{cm}$ in the fish. Using this value and the results

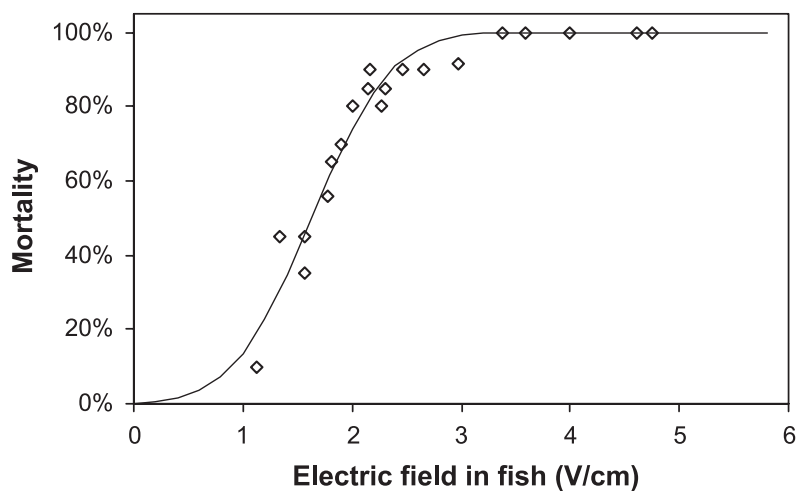

Fig. 4. Mortality in groups of fish following a 30-s exposure to an electric field in water of various conductivities. Electric field in fish is derived by data fitting. Solid line shows best fit cumulative Gaussian curve (mean=1.63 V/cm, standard deviation $=0.575 \mathrm{~V} / \mathrm{cm})$. 
Table 1

Electric field in water required to generate permanent insensibility in portion size rainbow trout. Information derived from experimental data. The frequency of the electrical power is $1000 \mathrm{~Hz}$ and the fish are perpendicular to field direction

\begin{tabular}{lrrrrrr}
\hline Water conductivity $(\mu \mathrm{S} / \mathrm{cm})$ & 50 & 100 & 160 & 300 & 500 & 1000 \\
Electric field $(\mathrm{V} / \mathrm{cm})$ 30-s exposure & 5.4 & 4.4 & 3.4 & 3.2 & 2.9 & 2.3 \\
Electric field $(\mathrm{V} / \mathrm{cm})$ 60-s exposure & 4.3 & 3.5 & 2.7 & 2.5 & 2.3 & 1.8 \\
\hline
\end{tabular}

given in Fig. 3, the electric field requirements for a 30-s exposure can be estimated for a range of water conductivities. These are given in Table 1. Comparison of these values with the results given in Appendix A shows agreement at each point.

Data in Appendix A relating to a 60-s exposure does not have a sufficient spread in mortality to permit the above analysis to be repeated for this exposure duration. However earlier work (Lines et al. 2003) indicated that an electric field of $2.5 \mathrm{~V} / \mathrm{cm}$ was necessary to generate permanent insensibility in trout exposed to the electric field for $60 \mathrm{~s}$ in water of conductivity $300-500 \mu \mathrm{S} / \mathrm{cm}$. The ratios in Fig. 3 can be applied to this $2.5 \mathrm{~V} / \mathrm{cm}$ electric field. This indicates a requirement for an electric field in the fish of $2.65 \mathrm{~V} / \mathrm{cm}$ and electric fields in water at various conductivities as indicated in Table 1 . These values have been derived without reference to the 60-s exposure experimental results given in Appendix A. However, comparison of these two sets of values shows complete agreement.

The experimental results for a 15 -s exposure given in the appendix do not identify any electric fields, which result in complete mortality, and the spread of mortality levels is again insufficient to determine the normal distribution and hence to predict the electric field corresponding to the three standard deviation point. The highest effective electric field in these fish is $4.6 \mathrm{~V} / \mathrm{cm}$. This occurs with water conductivity of $168 \mu \mathrm{S} / \mathrm{cm}$ and results in a mortality of $95 \%$. We suggest that this field might therefore represent the two standard deviation point. Assuming that that the relationship between the mean and the standard deviation of the electric field required to generate insensibility varies little with exposure duration, we tentatively estimate that an electric field of $5.5 \mathrm{~V} / \mathrm{cm}$ is required to achieve permanent insensibility in all trout. The electric fields required for a $15-\mathrm{s}$ exposure in water of various conductivities might therefore be expected to be around $160 \%$ of those identified in Table 1 for a 30-s exposure.

If the electric field values given in Table 1 are used to set up humane slaughter equipment, it is important to bear the limitations of the experiment in mind. It is possible that other factors such as stress, water temperature, and fish orientation will affect the electric field requirements. Adjustments should therefore be made to the electric field in the water if observation of the stunned fish suggests that permanent insensibility is not being achieved.

\section{Construction of an electric field model}

A mathematical model of the flow of electricity through the water and the fish head was constructed to generate a better understanding of the effect of water conductivity. Construction of this model was motivated by a wish to reduce the amount of 


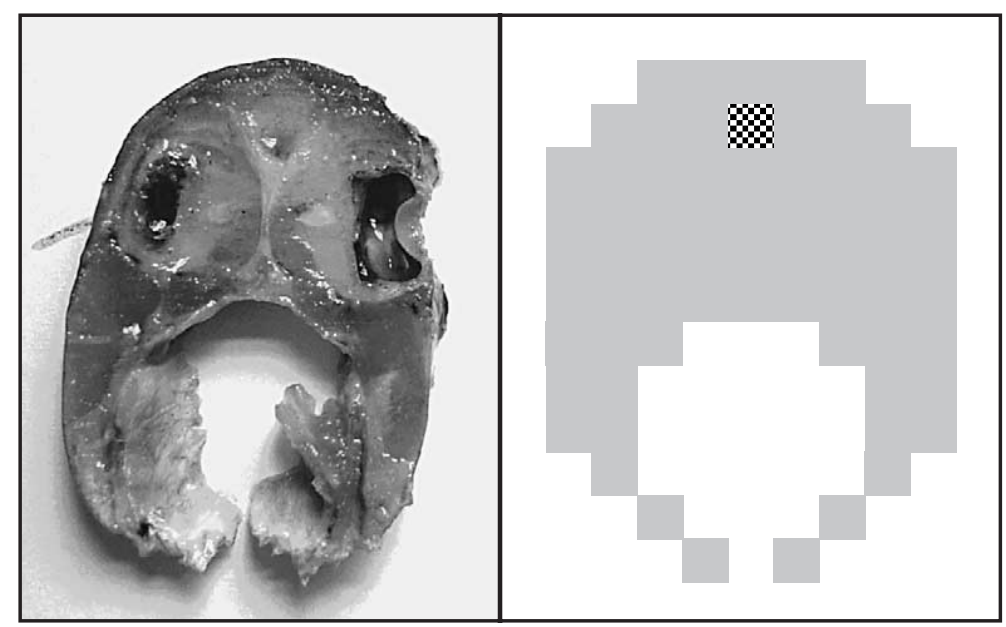

Fig. 5. Cross-section of trout head (cut through eyes) and model shape built from 3-mm cubes showing nominal location of brain for which the electric field is calculated.

experimentation needed to select suitable electric fields, to predict the electrical requirements for stunning fish at a wider range of water conductivities and to investigate how fish size, packing density and arrangement might be expected to affect the electric field requirement.

The model calculates the flow of electrical current through a simple two-dimensional representation of the fish head, which is immersed in water of a known conductivity. It assumes that the conductivity of the fish head is uniform, that it has a cross section as shown in Fig. 5 and that the situation can be modelled as a two-dimensional structure. The representation of the head in the model comprises an array of $3 \mathrm{~mm}$ cubes in a plane. Each is assigned an electrical resistance in both the horizontal and vertical direction, as determined by the measured conductivity of the water or the fish. The electric field is then calculated iteratively using a relaxation technique and applying Kirchoffs laws.

\section{Modelled results}

\subsection{Results for a single fish}

The modelling approach has been applied to a single fish placed centrally in a tank of water. Fig. 6 shows the relationship between the calculated electric field at the location of the brain of the fish, expressed as a proportion of the nominal electric field in the water (voltage difference between electrodes/distance between electrodes). These results indicate that at low water conductivities such as $50 \mu \mathrm{S} / \mathrm{cm}$ the electric field in the fish is half of the nominal electric field, while at high conductivities (e.g. $800 \mu \mathrm{S} / \mathrm{cm}$ ) the electric field is $50 \%$ greater than the nominal field. In sea water (conductivity, $50,000 \mu \mathrm{S} / \mathrm{cm}$ ), the model indicates that the electric field in the fish will be twice the nominal field strength. The experimentally determined results shown in Fig. 3 are overlaid for comparison. The 


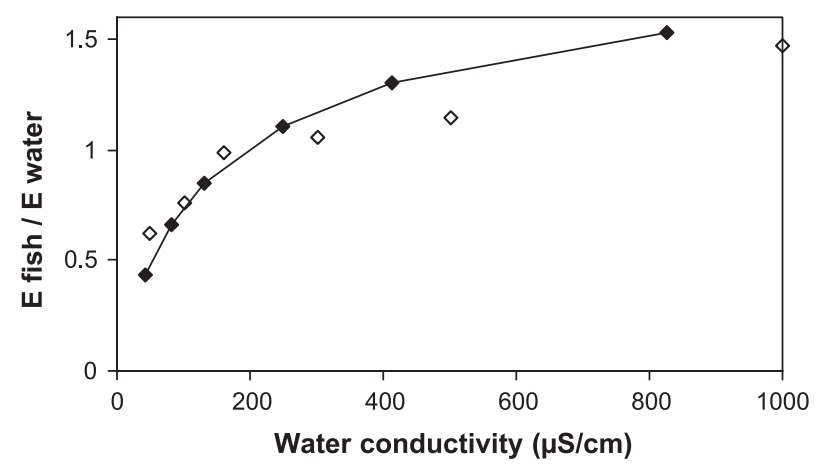

Fig. 6. Modelled relationship between electric field in the fish head and electric field in the water (solid line and points). Open diamonds show points estimated by data fitting for comparison.

derivation of these two sets of data is entirely separate except for the requirement that each curve has a value of unity at a water conductivity of $190 \mu \mathrm{S} / \mathrm{cm}$. The model predicts a slightly greater variation in the ratio between the field in the water and the field in the fish than is indicated by the experimental results. Some part of the increased variation can be attributed to the two-dimensional nature of the model. If the electrical current was not constrained to flow in a single plane, a greater range of current paths would be available which would reduce the variation in electric field strength. The degree to which this affects the model results in not known.

The model results have been applied to the experimental data presented in Fig. 1. The electric field in the fish is calculated from the measured electric field in the water and the water conductivity using the simulation results given in Fig. 6. The resulting relationship between fish mortality and electric field is shown in Fig. 7. It shows a progressive increase in fish mortality with the electric field, with a reasonably well defined mortality rate associated with each value of electric field. A cumulative Gaussian curve fitted to these data points to minimise the sum square deviation suggests that the mean electric field in

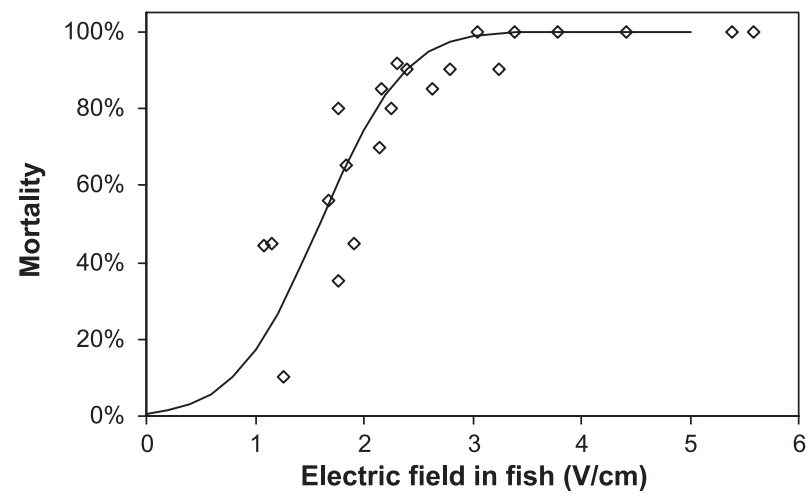

Fig. 7. Mortality in groups of fish following 30-s exposure to electric field in water of various conductivities. Electric field in fish is calculated using model. Solid line shows best fit cumulative Gaussian curve (mean=1.54 $\mathrm{V} / \mathrm{cm}$, standard deviation $=0.63 \mathrm{~V} / \mathrm{cm})$. 
the fish resulting in permanent insensibility is $1.54 \mathrm{~V} / \mathrm{cm}$ and that the standard deviation of this distribution is $0.63 \mathrm{~V} / \mathrm{cm}$. This suggests that a $3.5 \mathrm{~V} / \mathrm{cm}$ electric field in the fish would result in permanent insensibility after a 30 -s exposure. This is close to the value of $3.36 \mathrm{~V} /$ $\mathrm{cm}$ derived directly from the experimental data.

The data from this simulation can be used to predict the required electric field in the water for a range of water conductivities. Electric fields applicable to a 30-s exposure are derived from the requirement for an electric field in the fish of $3.5 \mathrm{~V} / \mathrm{cm}$, while those for a 60 -s exposure are derived, as before, from the observation that trout stunned in $300 \mu \mathrm{S} / \mathrm{cm}$ water require a field in the water of $2.5 \mathrm{~V} / \mathrm{cm}$. These electric fields are given in Table 2.

Comparison of the results in Tables 1 and 2 shows similarity for all water conductivities except $50 \mu \mathrm{S} / \mathrm{cm}$, where the simulation indicates values which are about $50 \%$ greater that the experimental data. Comparison of these simulation results with the direct experimental data for a 30-s exposure given in the appendix again shows agreement at all conductivities except in water of conductivity $50 \mu \mathrm{S} / \mathrm{cm}$.

\subsection{The effect of fish location, size and orientation}

The effect of the location in the tank on the electric field in the fish has been investigated using this simulation. Simulations used high $(825 \mu \mathrm{S} / \mathrm{cm})$ and low $(40 \mu \mathrm{S} / \mathrm{cm})$ conductivity fresh water and also sea water of conductivity $50,000 \mu \mathrm{S} / \mathrm{cm}$. Seawater conductivity however clearly extends the simulation beyond validated bounds, so must be considered indicative only. Deviations in the electric field at conductivities between 40 and $825 \mu \mathrm{S} / \mathrm{cm}$ would be expected to be smaller than those calculated for 40 and $825 \mu \mathrm{S} / \mathrm{cm}$. The model fish was placed centrally in the simulated tank, then moved progressively up towards the free water surface or laterally towards an electrode. The results given in Fig. 8 represent the deviation of the electric field in the fish from that which occurs in a fish placed centrally in the tank. This value is plotted against the minimum distance between the fish model and the electrode or water surface.

These results indicate that contact with, or close proximity to, the electrodes is likely to result in only small changes to the electric field in the fish. This is raised in low conductivity water and reduced in high conductivity water, but does not differ from the field in the centre of the tank by more than $20 \%$. At low water conductivities, proximity to the water surface also results in a small decrease in electric field in the fish. At high water conductivities proximity to the surface may result in a significant increase in electric field. Caution is however needed in the interpretation of this information since the twodimensional model probably over emphasises this increase. Since the only large change in

Table 2

The electric field in water required to generate permanent insensibility in portion size rainbow troutexperimental data interpreted using the simulation results (Fig. 6). The frequency of the electrical power is 1000 $\mathrm{Hz}$ and the fish oriented perpendicular to field direction

\begin{tabular}{lrrrrrr}
\hline Water conductivity $(\mu \mathrm{S} / \mathrm{cm})$ & 50 & 100 & 160 & 300 & 500 & 1000 \\
Electric field $(\mathrm{V} / \mathrm{cm})$ 30-s exposure & 7.5 & 4.9 & 3.8 & 2.9 & 2.5 & 2.1 \\
Electric field $(\mathrm{V} / \mathrm{cm})$ 60-s exposure & 6.5 & 4.2 & 3.3 & 2.5 & 2.1 & 1.8 \\
\hline
\end{tabular}



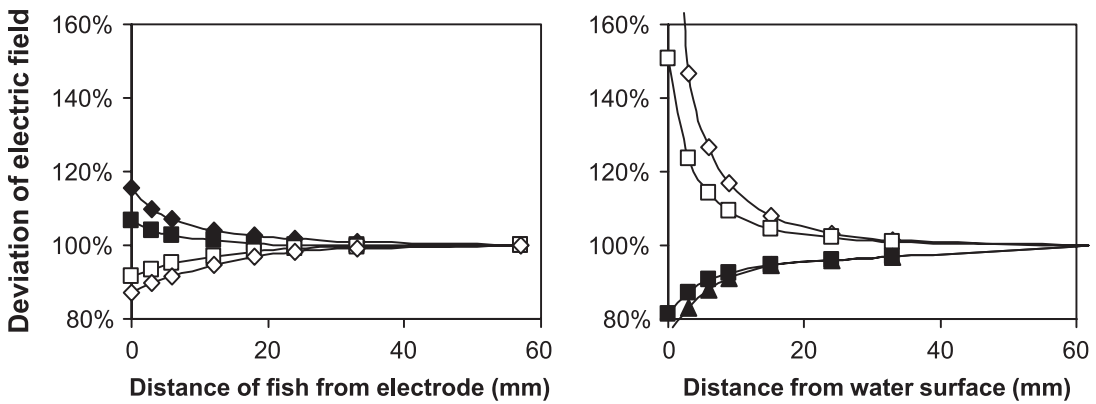

Fig. 8. Modelled results showing the effect of fish position on the electric field in fish. The electric field is given as a proportion of the field which occurs when the fish is in the centre of the tank and the distances are measured from the nearest point of the fish to the water surface or vertical electrode. Values are given for water with a conductivity of $40 \mu \mathrm{S} / \mathrm{cm}$ (solid diamond), $80 \mu \mathrm{S} / \mathrm{cm}$ (solid square), $825 \mu \mathrm{S} / \mathrm{cm}$ (open square) and for sea water of conductivity $50,000 \mu \mathrm{S} / \mathrm{cm}$ (open diamond).

electric field which occurs as a result of fish position is an increase, it is unlikely that variations in fish position will result in a fish being insufficiently stunned.

The effect of fish size was investigated by replacing each cell in the model fish with four cells, resulting in a model with the linear dimensions doubled and the notional fish mass increase by a factor of 8 . The electric field in the fish in water of $825 \mu \mathrm{S} / \mathrm{cm}$ was only $6 \%$ lower that in the original fish model. In low conductivity water $(40 \mu \mathrm{S} / \mathrm{cm})$, the electric field is higher than the original by a similar proportion. This suggests that any differences in the electric fields required to stun fish of different sizes, which might be observed are not simply due to the physical volume of the fish.

The fish was also modelled rotated by $90^{\circ}$ to represent a fish on its side. In this position, the brain was subjected to an electric field $15 \%$ greater than that of the upright fish in water of conductivity $825 \mu \mathrm{S} / \mathrm{cm}$ and $15 \%$ lower than the upright fish at low water conductivity. This variation in fish posture is therefore also unlikely to significantly influence effectiveness of the stunning process.

\subsection{Multiple fish in tank}

The simulation has also been used to investigate the effect on the electric field in the fish of the density and arrangement of fish in a tank by examining the six sample arrangements shown in Fig. 9. These fish are modelled in a $15 \times 15 \mathrm{~cm}$ cross-section stunning tank with electrodes extending the full height of the vertical tank walls. The resulting electric fields in the fish averaged over all the fish in the tank are given in Fig. 10, together with the results for a single fish for comparison. Error bars indicate the total range of electric fields for all arrangements. This indicates that the mean electric field experienced by a group of fish is lower than that experienced by a single fish in high conductivity water, and greater than that experienced by a single fish in low conductivity water. It also suggests that if the fish are well spaced apart, the electric field is likely to be similar to a single fish, whereas if they are held at high density in the tank or form a chain substantially across the tank from one electrode to the other, that they may experience an 

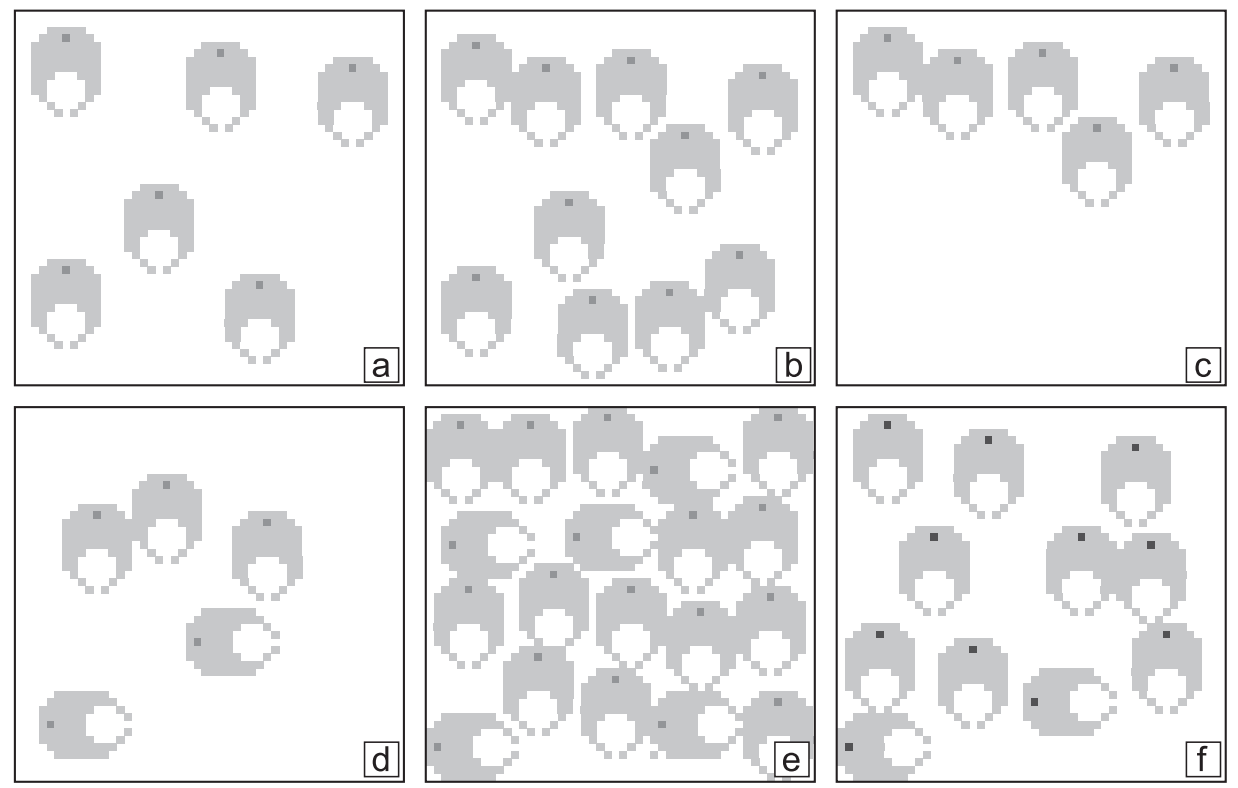

Fig. 9. Modelled arrangements of fish in tank. The tank was $15 \mathrm{~cm}$ wide and deep and the electrodes covered the full height of the vertical side walls.

electric field up to $15 \%$ lower than the single fish at high water conductivities or as much as $50 \%$ greater at low water conductivities. It is of interest to note that the experimentally derived results are a better match to the simulations of groups of fish than to the single fish. These experimental results were obtained from groups of five fish stunned in a tank the same size as that used in the simulation.

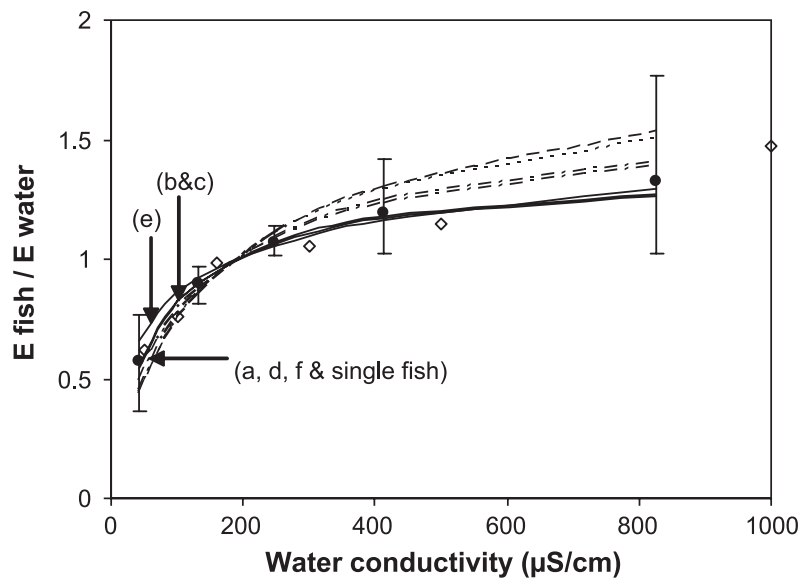

Fig. 10. Modelling results: the mean electric field in fish in the arrangements shown in Fig. 9. Single fish (dashed line), fish in arrangement (a) (dotted line), arrangements (d, f) (dot-dash line), arrangements (b, c, e) (solid lines). Solid point marker and error bars show mean, maximum and minimum observed electric fields for all fish in arrangements (a)-(f); open diamonds show points estimated by fitting experimental data. 
The range of electric fields experienced by individual fish increases with the difference between the fish and water conductivities. The largest positive deviations in electric field occurred at low water conductivity where the maximum electric field was $175 \%$ that which was experienced by the single fish. The largest negative deviation in electric field occurred at high water conductivity, where the electric field was $66 \%$ that which was experienced by the single fish. The variation in electric fields experience by individual fish appears to be related to the clustering of the fish and not directly to the density of the fish in the tank. The variation is greatest in arrangement (e) followed closely by arrangement (c). The variation in arrangement (a) is the lowest and only a third that of arrangement (e). Since the minimum electric field experienced in the tank should be used to define the electric field requirements, this increases the electric field requirement in both high and low conductivity water above that required to achieve the required mean electric field. It may indicate that at high fish densities and at water conductivities above $200 \mu \mathrm{S} / \mathrm{cm}$ the electric field in the fish should be assumed to equal the field in the water.

Extrapolation of these variations to sea water (conductivity $50,000 \mu \mathrm{S} / \mathrm{cm}$ ) suggests that the variation between the electric fields to which individual fish in a tank are exposed may be large. Simulation of fish arrangement (e) in sea water predicts that while the mean electric field in the fish will be twice that in the water, the minimum value for an individual fish will be only 0.2 that of the water and the maximum will be four times that of the water. These variations are however almost certainly an exaggeration of the truth since alternative current paths around and between the fish will occur in reality. If arrangement (e) is slightly modified to provide a small gap between each fish, then the mean electric field in the fish reduces to 1.4 that of the water and the minimum electric field increases from 0.2 to 1 .

\section{Discussion}

The fit of transformed data to a normal distribution curve shown in Fig. 4 and the similarity between the simulation results and the experimental results (Fig. 6) support the interpretation of the interaction between the fish and the electric field in the water which is proposed in this paper and used as the basis of the model. Examination of the various simulated curves in Fig. 10 indicates that the differences between the experimentally determined and predicted relationships between electric fields in the fish and in the water (Fig. 6) may be, in part, due to the number of fish in the tank because the simulation was based on a single fish, while the experimental work used groups of five fish.

The model used in this investigation could be improved by changing to a threedimensional representation, by increasing the spatial resolution and by including the electrical conductivity of the various components of the fish head rather than assuming it of uniform conductivity. These improvements would probably improve the predictive power of the model, particularly in high and low conductivity waters at the expense of simplicity and speed. The limitations on the model are likely to be greatest when attempting to model fish in sea water. The ability to make predictions in this environment would be a first step to making predictions of the electric field requirements for stunning a 
range of fish species of diverse shapes. Since the predicted electric field in the fish refers to a fish cross section of uniform conductivity, the predicted electric field in the brain must be considered a notional value and not the electric field value in the brain that might be measured directly.

The measured conductivity of the trout is lower than was expected. No publications appear to be available which identify conductivity measurements of fish tissue at the frequencies being used. Information on the electrical conductivity of human tissue is however presented by Gabriel et al. (1996a,b). This is also currently also available on an internet page published by the Italian National Research Council. This information indicates conductivity values of $200 \mu \mathrm{S} / \mathrm{cm}$ for fat, $3200 \mu \mathrm{S} / \mathrm{cm}$ for muscle and 15,000 $\mathrm{SS} / \mathrm{cm}$ for body fluids. Since the fish body is composed largely of fat, muscle and body fluids, a conductivity higher than $200 \mu \mathrm{S} / \mathrm{cm}$ might be expected. The experimental approach to measuring conductivity resulted in a small range of conductivities; however, variation of the fish conductivity in the simulation on this scale does not significantly affect the results or conclusions reached. A fish of uniform conductivity is clearly a simplification of the truth since different tissues have differing electrical conductivities. In particular, it was noted during experiments that the conductivity between the fish eyes appeared to be greater than elsewhere on the fish body.

Comparison of the electrical stun parameters required to achieve permanent insensibility with those identified by Robb and Roth (2003), reveals an interesting contrast. Robb and Roth investigated the stunning of salmon in seawater using an electric field running parallel to the long axis of the fish. A mortality rate of $45 \%$ was observed following exposure to an electric field of $0.25 \mathrm{~V} / \mathrm{cm}$ for 12 -s exposure. This suggests that the salmon in this situation are much more susceptible to electric stunning than the trout reported in this paper. A possible cause of this apparent difference is the direction of the electric field since the salmon were exposed to an electric field running 'head to tail' rather than 'side to side'. The difference may also reflect a difference in the resilience between salmon and trout. It may also be an indication that extrapolation of the simulation results to sea water conductivity is not valid.

\section{Conclusions}

A method for estimating the electric field in the fish head which is required to create permanent insensibility in fish is demonstrated. This enables the electrical field for stunning the fish to be defined without reference to the conductivity of the water.

Experimental results indicate that permanent insensibility in portion size rainbow trout can be achieve with electric field in the fish head of $2.6 \mathrm{~V} / \mathrm{cm}$ for a $60-\mathrm{s}$ duration, $3.4 \mathrm{~V} / \mathrm{cm}$ for a 30 -s duration or (tentatively) $5.5 \mathrm{~V} / \mathrm{cm}$ for a 15 -s duration.

In low conductivity water, the electric field generated in the fish head can be as little as half that in the water, while in high conductivity water the electric field generated in the fish head will be greater than that in the water.

A simulation model is described that provides a general technique for predicting the electric field, which must be supplied to the water to achieve the required electric field in the fish head. It is based only on the geometry and electrical conductivity of the fish and 
water. A higher resolution, three-dimensional model could be expected to produce more reliable results particularly at very high and very low water conductivities.

The simulation has been used to explore the effect on the electric field in the fish head for various positions in the tank, two orientations and two fish sizes. These variations are unlikely to result in significant changes in the electric field in the fish head.

The simulation has also been used to explore the effect on the electric field in the fish which are clustered in different ways. This suggests that the variation in the electric fields, which exist in individual fish in high density clusters, may be significant at high and low water conductivities. A consequence of this will be that in sea water the electric field required to generate permanent insensibility in all the fish in a cluster may be greater than that required for an isolated fish.

\section{Acknowledgement}

This work was funded by the British Trout Association, the Humane Slaughter Association and the BBSRC.

\section{Appendix A. Experimental data}

\begin{tabular}{|c|c|c|c|c|}
\hline $\begin{array}{l}\text { Exposure duration } \\
\text { (s) }\end{array}$ & $\begin{array}{l}\text { Water conductivity } \\
(\mu \mathrm{S} / \mathrm{cm})\end{array}$ & $\begin{array}{l}\text { Electric field } \\
(\mathrm{V} / \mathrm{cm})\end{array}$ & \# fish & Fish mortality \\
\hline 15 & 52 & 6.33 & 20 & $95 \%$ \\
\hline 15 & 104 & 4.67 & 20 & $85 \%$ \\
\hline 15 & 168 & 4.67 & 20 & $95 \%$ \\
\hline 15 & 979 & 2.33 & 40 & $98 \%$ \\
\hline 30 & 50 & 2.33 & 9 & $44 \%$ \\
\hline 30 & 55 & 3.58 & 20 & $80 \%$ \\
\hline 30 & 55 & 4.67 & 12 & $92 \%$ \\
\hline 30 & 53 & 6.33 & 20 & $100 \%$ \\
\hline 30 & 71 & 1.98 & 20 & $45 \%$ \\
\hline 30 & 100 & 2.33 & 9 & $56 \%$ \\
\hline 30 & 105 & 4.60 & 23 & $100 \%$ \\
\hline 30 & 161 & 2.33 & 20 & $85 \%$ \\
\hline 30 & 168 & 4.67 & 21 & $100 \%$ \\
\hline 30 & 195 & 1.81 & 20 & $65 \%$ \\
\hline 30 & 288 & 1.06 & 20 & $10 \%$ \\
\hline 30 & 285 & 1.92 & 20 & $80 \%$ \\
\hline 30 & 300 & 2.33 & 20 & $90 \%$ \\
\hline 30 & 297 & 4.50 & 40 & $100 \%$ \\
\hline 30 & 533 & 1.33 & 20 & $45 \%$ \\
\hline 30 & 544 & 1.83 & 20 & $85 \%$ \\
\hline 30 & 480 & 2.33 & 20 & $90 \%$ \\
\hline 30 & 947 & 1.09 & 20 & $35 \%$ \\
\hline 30 & 914 & 1.33 & 20 & $70 \%$ \\
\hline 30 & 983 & 1.47 & 20 & $90 \%$ \\
\hline
\end{tabular}


Appendix A (continued)

\begin{tabular}{lllll}
\hline $\begin{array}{l}\text { Exposure duration } \\
(\mathrm{s})\end{array}$ & $\begin{array}{l}\text { Water conductivity } \\
(\mu \mathrm{S} / \mathrm{cm})\end{array}$ & $\begin{array}{l}\text { Electric field } \\
(\mathrm{V} / \mathrm{cm})\end{array}$ & \# fish & Fish mortality \\
\hline 30 & 955 & 2.33 & 20 & $100 \%$ \\
60 & 55 & 4.67 & 40 & $100 \%$ \\
60 & 101 & 4.67 & 6 & $100 \%$ \\
60 & 163 & 2.33 & 20 & $95 \%$ \\
60 & 291 & 2.33 & 40 & $100 \%$ \\
60 & 487 & 2.33 & 20 & $100 \%$ \\
60 & 903 & 1.33 & 25 & $96 \%$ \\
\hline
\end{tabular}

\section{References}

Gabriel, C., Gabriel, S., Corthout, E., 1996a. The dielectric properties of biological tissues: I. Literature survey. Physics in Medicine and Biology 41, 2231-2249.

Gabriel, S., Lau, R.W., Gabriel, C., 1996b. The dielectric properties of biological tissues: II. Measurements in the frequency range $10 \mathrm{~Hz}$ to $20 \mathrm{GHz}$. Physics in Medicine and Biology 41 (1996), 2251-2269.

Italian National Research Council. http://safeemf.iroe.fi.cnr.it/tissprop/htmlclie/htmlclie.htm.

Kestin, S.C., van de Vis, J.W., Robb, D.H.F., 2002. Protocol for assessing brain function in fish and the effectiveness of methods used to stun and kill them. Veterinary Record 150, 302-307.

Lamarque, P., 1990. Electrophysiology of fish in electric fields. In: Cowx, I.G., Lamarque, P. (Eds.), Fishing with electricity: applications in freshwater fisheries management. Fishing News Books, London.

Lines, J.A., Kestin, S.C., 2004a. Trout slaughter-time for a change. Trout News 37, 13-16. http:// www.cefas.co.uk/publications/troutnews/tnews37.pdf.

Lines, J.A., Kestin, S.C., 2004b. Electric stunning of trout: power reduction using a two stage stun. Aquacultural Engineering (accepted for publication).

Lines, J.A., Robb, D.H., Kestin, S.C., Crook, S.C., Benson, T., 2003. Electric stunning: a humane slaughter method for trout. Aquacultural Engineering 28, 141-154.

McMichael, G.A., 1993. Examination of electrofishing injury and short term mortality in hatchery rainbow trout. North American Journal of Fisheries Management 13, 229-233.

Robb, D.H.F., Kestin, S.C., 2002. Methods used to kill fish: field observations and literature reviewed. Animal Welfare 11, 269-282.

Robb, D.H.F., Roth, B., 2003. Brain activity of Atlantic salmon (Salmo salar) following electrical stunning using various field strengths and pulse durations. Aquaculture 216, 363-369.

Robb, D.H.F., O’Callaghan, M.O., Lines, J.A., Kestin, S.C., 2002. Electrical stunning of rainbow trout (Oncorhynchus mykiss): factors that affect stun duration. Aquaculture 205, 359-371.

Roth, B., Imseland, S., Moeller, D., Slinde, E., 2003. Effect of electric stunning and current duration on stunning and injuries in market sized Atlantic salmon held in sea water. North American Journal of Aquaculture 65, 8-13.

Sharber, N.G., Carothers, S.W., 1990. Influence of electric fishing pulse shape on spinal injuries in adult rainbow trout. In: Cox, I.G. (Ed.), Developments in Electric Fishing. Fishing News Books, London.

Spencer, S.L., 1967. Internal injuries of large moth bass and bluegills caused by electricity. Progressive Fish Culturist 29, 168-169. 